\title{
Efficacy and tolerance of osilodrostat in patients with severe Cushing's syndrome due to non-pituitary cancers
}

\author{
Magalie Haissaguerre, Marie Puerto, Marie-Laure Nunes and Antoine Tabarin \\ Department of Endocrinology, Diabetes and Nutrition, Hôpital Haut Lévêque, CHU and University of Bordeaux, \\ Pessac, France
}

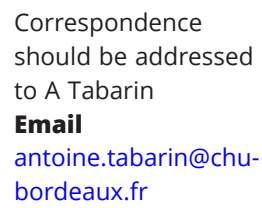

\section{To the editor}

Severe hypercortisolism (SH) is a rare life-threatening endocrine emergency, mainly caused by ectopic ACTH syndrome (EAS), which requires a rapid normalization of cortisol concentrations $(1,2)$. Due to their rapidity of action, steroidogenesis inhibitors, such as ketoconazole and metyrapone, given in monotherapy or in association, are recommended in this situation $(1,3,4)$. Osilodrostat is a recently developed inhibitor of adrenal 11B-hydroxylase (5) which has been shown to be effective, using a progressive titration of the drug dosage, in the treatment of Cushing's disease $(5,6,7)$. Limited data are available on the use of osilodrostat in SH induced by EAS (8). Following the shortage of metyrapone in 2019, a temporary authorization for the use of osilodrostat was given by French authorities. We report herein our experience with osilodrostat in the treatment of three patients with severe Cushing's syndrome due to EAS and adrenal carcinoma (ACC).

\section{Patient 1}

A 51-year-old woman presented with a small cell lung carcinoma (SCLC) responsible for SH. She was effectively treated for 2 years by chemotherapy and $500 \mathrm{mg} /$ day metyrapone after developing liver intolerance to ketoconazole. Following metyrapone shortage, $2 \mathrm{mg}$ /day osilodrostat allowed control of cortisol levels. Recurrence of the SCLC was associated with an increase in plasma cortisol to $1200 \mathrm{nmol} / \mathrm{L}$. Osilodrostat dose was immediately increased to $5 \mathrm{mg} /$ day for 4 days, and based on repeated plasma cortisol measurements, it was increased every 2-4 days in $5 \mathrm{mg} /$ day steps. A maximal dose of $25 \mathrm{mg} /$ day was reached after 2 weeks which allowed the mean plasma cortisol concentrations (determined from 3-day time samples) to be in the target range (200-500 nmol/L). Control of SH was sustained for 3 months until the death of the patient due to tumoral burden (Fig. 1A).

\section{Patient 2}

A 39-year-old man presented with an ACC responsible for SH (plasma cortisol $=1495 \mathrm{nmol} / \mathrm{L}$ ). Liver intolerance occurred after 10 days of ketoconazole treatment at $1200 \mathrm{mg} /$ day. Osilodrostat was introduced at $5 \mathrm{mg} /$ day together with mitotane $1500 \mathrm{mg} /$ day. Based on repeated plasma cortisol measurements, osilodrostat was increased every $4-5$ days by $4-5 \mathrm{mg}$ /day steps. The maximal dosage of $44 \mathrm{mg} /$ day was reached after 2 weeks and achieved normalization of plasma cortisol. To simplify anticortisolic treatment during neoadjuvant anticancer chemotherapy, a block-and-replace (BAR) regimen was employed with osilodrostat $30 \mathrm{mg} /$ day associated with hydrocortisone that was introduced when 08:00 h plasma cortisol started to decrease below normal $(173 \mathrm{nmol} / \mathrm{L})$. A high dosage (60 mg/day) of hydrocortisone was prescribed as required in patients treated with mitotane (9). Hypercortisolism was controlled for 4 months until surgical resection of the ACC (Fig. 1B). 
A
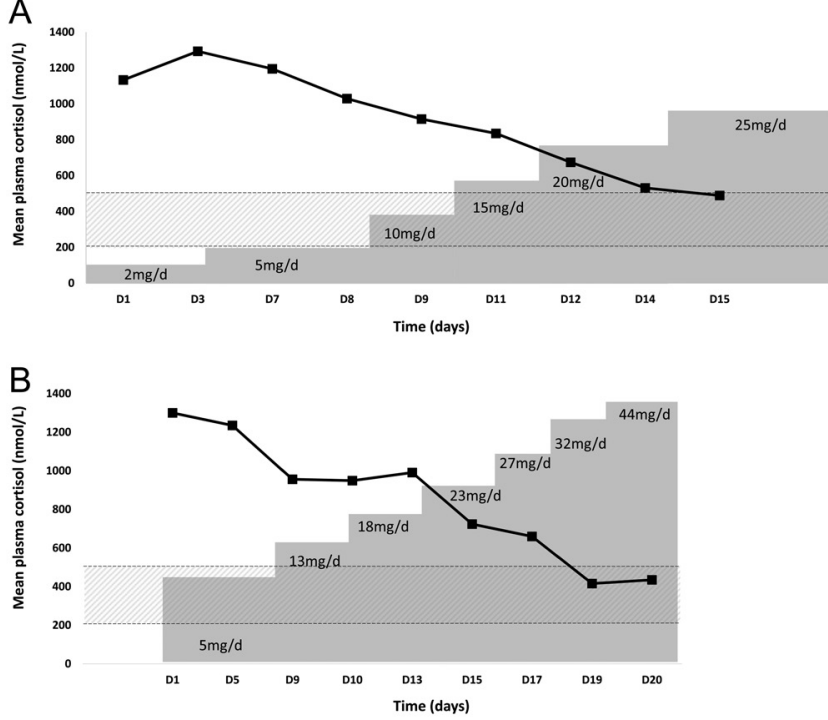

C

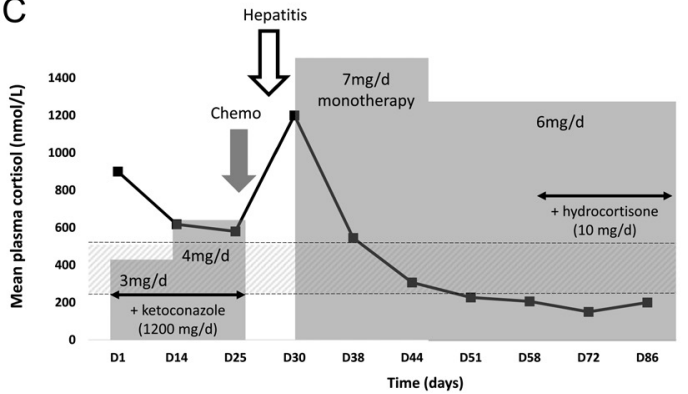

Figure 1

Evolution of mean plasma cortisol concentrations in three patients treated with osilodrostat (A: patient 1; B: patient 2; C: patient 3). Cortisol values are the mean of 3-day time measurements. The shaded area corresponds to the normal range in our laboratory $(200-500 \mathrm{nmol} / \mathrm{L})$. The gray background steps represent the titration regimen of osilodrostat in mg/day. Arrow chemo: beginning of the second line antineoplastic chemotherapy. Arrow hepatitis: occurrence of liver intolerance to ketoconazole.

\section{Patient 3}

A 70-year-old man presented with a SCLC responsible for $\mathrm{SH}$ (plasma cortisol $=1970 \mathrm{nmol} / \mathrm{L}$ ) that was controlled with chemotherapy associated with metyrapone (750 mg/day) and ketoconazole (1200 mg/day). Following metyrapone shortage, osilodrostat was introduced at $1 \mathrm{mg} /$ day, a dose that maintained the control of hypercortisolism, in association with ketoconazole (1200 $\mathrm{mg}$ /day). Recurrence of the SCLC was associated with an increase in plasma cortisol to $900 \mathrm{nmol} / \mathrm{L}$. While ketoconazole treatment was continued, osilodrostat was increased to $4 \mathrm{mg} /$ day over 14 days, allowing a 50\% decrease in cortisol concentration. A pharmacological interaction between chemotherapy (paclitaxel) and ketoconazole induced liver intolerance and all medications were stopped. After 1 week of drug withdrawal, plasma cortisol increased to $1200 \mathrm{nmol} / \mathrm{L}$. Osilodrostat was reintroduced as monotherapy at $7 \mathrm{mg}$ /day leading to control of SH in 7 days. Three weeks later, due to low cortisol concentrations (150 nmol/L, normal: $200-500 \mathrm{nmol} / \mathrm{L}$ ), a $10 \mathrm{mg}$ morning dose of hydrocortisone was prescribed in association with osilodrostat at $6 \mathrm{mg} /$ day. This BAR regimen allowed reintroduction of anticancer chemotherapy and sustained control of hypercortisolism until the death of the patient 2 months later due to tumoral burden (Fig. 1C).

\section{Discussion}

These observations show that, using variable regimens, osilodrostat rapidly and safely controlled SH in three patients in whom metyrapone and ketoconazole could not be prescribed. The time to control of plasma cortisol concentrations was $\leq 2$ weeks, and a maximal dose of $44 \mathrm{mg} /$ day was used. The tolerance of osilodrostat was excellent: neither nausea nor changes in QTc or kalemia were observed. To date, we are only aware of one Japanese study (8) involving three patients with $\mathrm{SH}$ and EAS, who were treated in a phase 2 study using a fixed dosetitration protocol. Although the individual data were not provided, analysis of figures from this paper shows that osilodrostat normalized UFC in the short-term in approximately 5-9 weeks, that is, a significantly longer period than that observed in our patients. The precise dosage of osilodrostat was not specifically given, but in any case cannot be extrapolated to Caucasians, since the relative bioavailability of the drug is approximately $20 \%$ higher in Asian patients as compared to other ethnicities $(5,8)$.

In our series, osilodrostat controlled rapidly $\mathrm{SH}$ using a rapid increase in dosage that differs from that used in the Linc Studies $(5,6,7)$ and the Japanese study (8). Our titration protocol was established based on the pharmacokinetics of osilodrostat, including a rapid absorption (Tmax $\approx 1 \mathrm{~h}$ ), a half-life $\approx 4 \mathrm{~h}$, and a steady state reached in 2 days (5). These data associated with those derived from animal studies, suggesting a dramatic decrease in plasma cortisol after at most $8 \mathrm{~h}$ following a single dose (10), which prompted us to use 2-5 days titration steps based on serial plasma cortisol measurements. 
Osilodrostat was used using a BAR regimen in two patients, a therapeutic scenario which has not been previously described with this drug. The TID regimen and availability of various dosages for osilodrostat significantly reduced the number of steroidogenesis inhibitor pills to be taken $(3,4,11,12)$, a point that may favor patient compliance and be relevant in terms of quality of life. The BAR regimen may peculiarly be useful in a complex situation, such as $\mathrm{SH}$ in patients with non-pituitary cancers. Indeed, when antineoplastic therapy is effective on tumor burden, a condition that is usually evaluated by imaging after several weeks of delay, it may also induce a rapid concomitant decrease in cortisol which complicates the monitoring of drug dosage and requires repeated cortisol measurements. In our opinion, the BAR regimen with an effective drug that is associated with a low prevalence of drug-to-drug interactions (5) represents a valid therapeutic approach in these patients.

In conclusion, in a block-and-replace strategy aiming at controlling SH rapidly (13), and based on a limited number of observations, we suggest to start osilodrostat at a relatively high dosage, of at least $6 \mathrm{mg} /$ day, when baseline cortisol levels are $>1000 \mathrm{nmol} / \mathrm{L}$; re-assess plasma cortisol (ideally associated with UFC) after 2-5 days for further adjustments and consider prescription of glucocorticoid replacement. Complementary observations in these complex therapeutic situations are needed to determine optimal starting doses of osilodrostat and duration of titration steps.

\section{Declaration of interest}

The authors declare that there is no conflict of interest that could be perceived as prejudicing the impartiality of this letter.

\section{Funding}

This work did not receive any specific grant from any funding agency in the public, commercial, or not-for-profit sector or industry.

\section{References}

1 Young J, Haissaguerre M, Viera-Pinto O, Chabre O, Baudin E \& Tabarin A. MANAGEMENT OF ENDOCRINE DISEASE: Cushing's syndrome due to ectopic ACTH secretion: an expert operational opinion. European Journal of Endocrinology 2020182 R29-R58. (https://doi.org/10.1530/EJE-19-0877)
2 Nieman LK, Biller BMK, Findling JW, Murad MH, Newell-Price J, Savage MO, Tabarin A \& Endocrine Society. Treatment of Cushing's syndrome: an Endocrine Society clinical practice guideline. Journal of Clinical Endocrinology and Metabolism 2015100 2807-2831. (https:// doi.org/10.1210/jc.2015-1818)

3 Daniel E \& Newell-Price JDC. Therapy of endocrine disease: steroidogenesis enzyme inhibitors in Cushing's syndrome. European Journal of Endocrinology 2015172 R263-R280. (https://doi. org/10.1530/EJE-14-1014)

4 Corcuff JB, Young J, Masquefa-Giraud P, Chanson P, Baudin E \& Tabarin A. Rapid control of severe neoplastic hypercortisolism with metyrapone and ketoconazole. European Journal of Endocrinology 2015 172 473-481. (https://doi.org/10.1530/EJE-14-0913)

5 Duggan S. Osilodrostat: first approval. Drugs $2020 \mathbf{8 0} 495-500$. (https://doi.org/10.1007/s40265-020-01277-0)

6 Bertagna X, Pivonello R, Fleseriu M, Zhang Y, Robinson P, Taylor A, Watson CE, Maldonado M, Hamrahian AH, Boscaro M et al. LCI699, a potent $11 \beta$-hydroxylase inhibitor, normalizes urinary cortisol in patients with Cushing's disease: results from a multicenter, proof-ofconcept study. Journal of Clinical Endocrinology and Metabolism 2014 99 1375-1383. (https://doi.org/10.1210/jc.2013-2117)

7 Fleseriu M, Pivonello R, Young J, Hamrahian AH, Molitch ME, Shimizu C, Tanaka T, Shimatsu A, White T, Hilliard A et al. Osilodrostat, a potent oral 11 $\beta$-hydroxylase inhibitor: 22 -week, prospective, Phase II study in Cushing's disease. Pituitary 201619 138-148. (https://doi.org/10.1007/s11102-015-0692-z)

8 Tanaka T, Satoh F, Ujihara M, Midorikawa S, Kaneko T, Takeda T, Suzuki A, Sato M \& Shimatsu A. A multicenter, phase 2 study to evaluate the efficacy and safety of osilodrostat, a new $11 \beta$-hydroxylase inhibitor, in Japanese patients with endogenous Cushing's syndrome other than Cushing's disease. Endocrine Journal 2020 In press. (https://doi.org/10.1507/endocrj.EJ19-0617)

9 Chortis V, Taylor AE, Schneider P, Tomlinson JW, Hughes BA, O’Neil DM, Libé R, Allolio B, Bertagna X, Bertherat J et al. Mitotane therapy in adrenocortical cancer induces CYP3A4 and inhibits $5 \alpha$-reductase, explaining the need for personalized glucocorticoid and androgen replacement. Journal of Clinical Endocrinology and Metabolism 201398 161-171. (https://doi.org/10.1210/jc.2012-2851)

10 Ménard J, Rigel DF, Watson C, Jeng AY, Fu F, Beil M, Liu J, Chen W, $\mathrm{Hu} \mathrm{CW}$, Leung-Chu J et al. Aldosterone synthase inhibition: cardiorenal protection in animal disease models and translation of hormonal effects to human subjects. Journal of Translational Medicine 201412 340. (https://doi.org/10.1186/s12967-014-0340-9)

11 Verhelst JA, Trainer PJ, Howlett TA, Perry L, Rees LH, Grossman AB, Wass JA \& Besser GM. Short and long-term responses to metyrapone in the medical management of 91 patients with Cushing's syndrome. Clinical Endocrinology 199135 169-178. (https://doi. org/10.1111/j.1365-2265.1991.tb03517.x)

12 Kamenický P, Droumaguet C, Salenave S, Blanchard A, Jublanc C, Gautier JF, Brailly-Tabard S, Leboulleux S, Schlumberger M, Baudin E et al. Mitotane, metyrapone, and ketoconazole combination therapy as an alternative to rescue adrenalectomy for severe ACTH-dependent Cushing's syndrome. Journal of Clinical Endocrinology and Metabolism 201196 2796-2804. (https://doi.org/10.1210/jc.2011-0536)

13 Newell-Price J, Nieman LK, Reincke M \& Tabarin A. ENDOCRINOLOGY IN THE TIME OF COVID-19: Management of Cushing's syndrome. European Journal of Endocrinology 2020183 G1-G7. (https://doi.org/10.1530/EJE-20-0352)

Received 29 May 2020

Revised version received 24 June 2020

Accepted 17 July 2020 